\title{
Dab1 Contributes to Angiotensin II-Induced Apoptosis via p38 Signaling Pathway in Podocytes
}

\author{
Zhao Gao, Xinghua Chen, Kai Zhu, Ping Zeng, and Guohua Ding \\ Division of Nephrology, Renmin Hospital of Wuhan University, 238 Jiefang Rd., Wuhan, Hubei 430060, China \\ Correspondence should be addressed to Guohua Ding; ghxding@gmail.com
}

Received 4 February 2017; Revised 16 April 2017; Accepted 10 May 2017; Published 5 June 2017

Academic Editor: Deepak Nihalani

Copyright (C) 2017 Zhao Gao et al. This is an open access article distributed under the Creative Commons Attribution License, which permits unrestricted use, distribution, and reproduction in any medium, provided the original work is properly cited.

\begin{abstract}
Numerous studies have found that angiotensin II (Ang II) participates in podocyte apoptosis and exacerbates progression of endstage kidney disease (ESKD). However, its underlying mechanism remains largely unexplored. As a homolog of Drosophila disabled (Dab) protein, Dabl plays a vital role in cytoskeleton, neuronal migration, and proliferation. In the present study, our data revealed that Ang II-infused rats developed hypertension, proteinuria, and podocyte injury accompanied by Dabl phosphorylation and increased reelin expression in kidney. Moreover, Ang II induced podocyte apoptosis in vitro. Dab1 phosphorylation and reelin expression in podocytes were increased after exposure to Ang II. Conversely, Dabl small interfering RNA (siRNA) exerted protective effects on Ang II-induced podocyte apoptosis, resulting in decreased p38 phosphorylation and reelin expression. These results indicated that Dab1 mediated Ang II-induced podocyte apoptosis via p38 signaling pathway.
\end{abstract}

\section{Introduction}

As terminally differentiated cells, podocytes play a crucial role in establishing the integrity and selective permeability of the glomerular filtration barrier [1-3]. Accumulating evidence has shown that podocyte injury is associated with proteinuria and several glomerular diseases [4-7]. As an important risk factor for the initiation and progression of chronic kidney diseases [8, 9], angiotensin II (Ang II) exerts hemodynamic effects on renal tissue and a direct effect on podocyte injury. Recent studies have shown that Ang II can induce podocyte apoptosis both in vivo and in vitro [10-12]. However, the exact molecular mechanism of Ang II-induced podocyte apoptosis remains largely unexplored.

Disabled-1 (Dab1), a homolog of the Drosophila disabled protein, consists of three main domains as follows: C-terminal serine/threonine-rich region, $\mathrm{N}$-terminal protein interaction/phosphotyrosine binding domain, and a tyrosine-rich region. Dabl located in cytoplasm is an adaptor protein associated with neuronal migration and polarization [13]. When reelin, a secreted extracellular matrix glycoprotein, or apoE binds to very low-density lipoprotein receptor (VLDLR) and/or apolipoprotein receptor 2 (ApoER2), Dab1 interacts with the SH2 domains of Src, Fyn, and Abl, leading to Dab1 tyrosine phosphorylation and activation of downstream signaling pathways, such as Crk-C3G-Rapl-cadherin pathway and PI3K-Akt pathway [14].

Our previous study has indicated that c-Abl upregulation promotes podocyte apoptosis upon exposure to Ang II via Akt signaling [12]. In the preexperiment, we found that Dab1 and reelin protein were expressed in rat and podocytes by western blotting and immunofluorescence assay. We hypothesized that Dabl participated in podocyte apoptosis and aimed to examine the role of Dabl in Ang II-induced podocyte apoptosis and signal transduction.

\section{Materials and Methods}

2.1. Animals. A total of 16 male specific-pathogen-free (SPF) Sprague-Dawley rats (weighing 110-140 g) were supplied by the Research Center of Medical Experimental Animals of Wuhan University. Animals were bred in an atmosphere with controlled temperature and humidity under an artificial light cycle. Food pellets and water were available ad libitum. Rats embedded with osmotic minipump (Alzet model 2002 or 2004, CA) were randomly subjected to normal saline 
infusion or Ang II infusion at a dose of $400 \mathrm{ng} / \mathrm{kg} / \mathrm{min}$ for 4 weeks. Tail cuff plethysmography was used to measure systolic blood pressure at different time points (weeks 1, 2, 3, and 4). Rats were individually placed into metabolic cages for $24 \mathrm{~h}$ to collect urine, and urinary albumin was determined at the above-mentioned time points (weeks 1, 2, 3 and 4). Urinary albumin was determined with a competitive ELISA kit (Shibayagi, Shibukawa, Japan). Animals were sacrificed after 4 weeks. Part of the kidney was fixed in $4 \%$ phosphatebuffered paraformaldehyde for pathological analysis of renal damage, and the rest was stored at $-80^{\circ} \mathrm{C}$ prior to further biochemical analysis. Glomeruli were isolated by $177 \mu \mathrm{m}$, $125 \mu \mathrm{m}$, and $74 \mu \mathrm{m}$ stainless steel sieves. The experimental procedures and protocols were approved by the Ethical Committee for the Experimental Use of Animals of Renmin Hospital of Wuhan University, Wuhan, Hubei Province, China.

2.2. Cell Culture. Conditionally immortalized mouse podocytes were kindly provided by Dr. Peter Mundel (Massachusetts General Hospital, Boston, MA). Podocytes were cultured in RPMI 1640 medium (HyClone, USA) supplemented with $10 \%$ heat-inactivated fetal calf serum (Gibco, USA), $100 \mathrm{U} / \mathrm{mL}$ penicillin $\mathrm{G}, 100 \mathrm{mg} / \mathrm{mL}$ streptomycin, and $10 \mathrm{U} / \mathrm{mL}$ recombinant murine interferon- $\gamma$ (Pepro Tech, Rocky Hill, NJ) at $33^{\circ} \mathrm{C}$. Proliferating podocytes were transferred to an incubator at $37^{\circ} \mathrm{C}$ for 7 days to induce differentiation without interferon. The differentiated cells were stimulated with Ang II (Enzo Life Sciences, Farmingdale, NY) for various durations.

2.3. Dabl siRNA Transfection. Dabl siRNA transfection was carried out according to the HiPerFect Transfection Reagent Handbook (QIAGEN, Germany). Four Dabl siRNAs (siRNA sequences: sil AAGGGAGAACACAAACAGAAA, si3 CAGCGAAGCCACTTTGATAAA, si4 CACTTTGATAAAGAGGTTTAA) were designed and synthesized by QIAGEN (Germany). Briefly, $2 \times 10^{5}$ cells were seeded in a 6-well plate and then transfected with a $100 \mu \mathrm{L}$ mixture containing 150 ng Dab1 siRNA (or a negative control with scrambled siRNA) and $12 \mu \mathrm{L}$ of HiPerFect transfection reagent for $48 \mathrm{~h}$.

2.4. Double Immunofluorescence Assay. The cell climbing film was fixed in $4 \%$ paraformaldehyde at $4^{\circ} \mathrm{C}$ for $30 \mathrm{~min}$ and then blocked in 5\% bovine serum albumin at room temperature for $30 \mathrm{~min}$. The films were, respectively, stained with the following antibodies: Dab1 antibody (1: 50, sc-13981, Santa Cruz Biotechnology), phospho-Dabl antibody (1:25, sc-133293, Santa Cruz Biotechnology), and reelin antibody (1:50, sc-25346, Santa Cruz Biotechnology) at $4^{\circ} \mathrm{C}$ overnight. Subsequently, films were incubated with FITC-conjugated IgG as the secondary antibody at $37^{\circ} \mathrm{C}$ for $45 \mathrm{~min}$ in the dark. The sections were observed under fluorescence microscope (Olympus, Japan).

Paraffin-embedded sections were deparaffinized and treated with $3 \% \mathrm{H}_{2} \mathrm{O}_{2}$ at room temperature for $30 \mathrm{~min}$. Antigen retrieval for Dabl, phospho-Dabl, and reelin was carried out in high-pressure citrate buffer (0.01 M, pH 6.0) for $10 \mathrm{~min}$. The sections were blocked with $5 \%$ bovine serum albumin for $30 \mathrm{~min}$, incubated with nephrin antibody (1:100, sc-32529, Santa Cruz Biotechnology), Dab1 antibody (1:50, sc-13981, Santa Cruz Biotechnology), phospho-Dab1 antibody (1:30, sc-133293, Santa Cruz Biotechnology), and reelin antibody (1:50, sc-25346, Santa Cruz Biotechnology) at $4^{\circ} \mathrm{C}$ overnight, and then stained with FITC-conjugated $\operatorname{IgG}$ at $37^{\circ} \mathrm{C}$ for $60 \mathrm{~min}$ in the dark. All microscopic images were recorded using a fluorescence microscope (Olympus, Japan).

2.5. Apoptosis Assay. Podocyte apoptosis in kidney tissue was assessed by TUNEL (Roche, Germany) according to the manufacturer's instructions. Briefly, the paraffin-embedded sections were dewaxed and incubated with $3 \% \mathrm{H}_{2} \mathrm{O}_{2}$ for $30 \mathrm{~min}$. Antigen retrieval was performed in high-pressure citrate buffer (0.01 M, pH 6.0) for $10 \mathrm{~min}$. Subsequently, the sections were blocked with $10 \%$ goat serum at room temperature for $30 \mathrm{~min}$ and incubated with terminal deoxynucleotidyl transferase (TdT) and digoxigenin-11-dUTP at room temperature for $1 \mathrm{~h}$. Next, sections were incubated with streptavidinbiotin-peroxidase-conjugated antidigoxigenin-11-dUTP antibody for $30 \mathrm{~min}$. The negative control was omitted TdT. The podocytes were counted in 3 glomerular cross sections using 10 randomly selected fields. Two consecutive sections were stained with PAS and TUNEL, respectively. The sections were scanned by automatic digital slice scanning system (Leica SCN400F, Germany). Podocytes were identified and counted in PAS section $[15,16]$. In the same view, apoptotic podocytes were counted in TUNEL section.

2.6. Western Blotting. Glomeruli and podocytes were lysed with RIPA buffer (Beyotime, China) containing protease inhibitor cocktail (Sigma-Aldrich) and PMSF (Beyotime, China) on the ice for $30 \mathrm{~min}$, and then the cell lysates were centrifuged at $12,000 \mathrm{rpm}$ for $10 \mathrm{~min}$ at $4^{\circ} \mathrm{C}$. Subsequently, the supernatants were mixed with $5 \mathrm{x}$ loading buffer and boiled at $100^{\circ} \mathrm{C}$ for $5 \mathrm{~min}$. The proteins were separated with SDS-PAGE and then electrotransferred onto PVDF membranes (Merck Millipore). The membranes were incubated with primary antibodies (rabbit polyclonal Dabl antibody, 1:50, Santa Cruz Biotechnology, Dallas, TX; rabbit polyclonal phospho-Dab1 antibody, 1:50, Santa Cruz Biotechnology, Dallas, TX; mouse monoclonal reelin antibody, 1:50, Santa Cruz Biotechnology, Dallas, TX; rabbit polyclonal p38 antibody, 1:1,000, Cell Signaling Technology; rabbit polyclonal phospho-p38 antibody, 1:1,000, Cell Signaling Technology; rabbit polyclonal ERK antibody, 1:1,000, Cell Signaling Technolog; rabbit polyclonal phospho-ERK antibody, 1:1,000, Cell Signaling Technology; rabbit polyclonal caspase-3 antibody, 1:1000, GeneTex Inc.; rabbit polyclonal bcl-2 antibody, $1: 1000$, GeneTex Inc.; rabbit polyclonal $\beta$-actin antibody, 1:1000, Antgene, Hubei, China) at $4^{\circ} \mathrm{C}$ overnight. An Alexa Fluor 790-labeled goat anti-rabbit/mouse IgG $(1: 30,000$; Jackson ImmunoResearch, USA) was used as secondary antibody, and the blots were visualized with a LI-COR Odyssey Infrared Imaging System. 


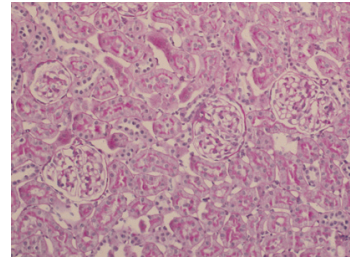

(A)

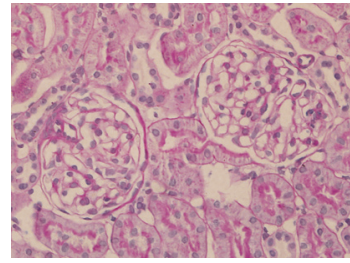

(C)

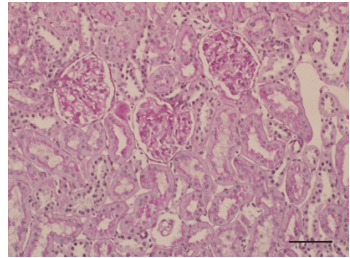

(B)

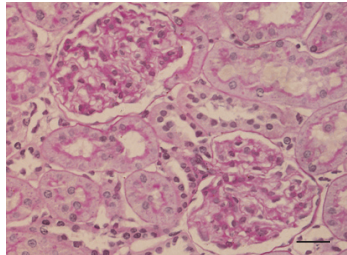

(D)

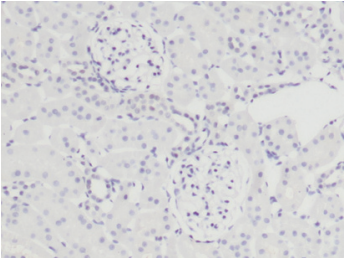

(A)

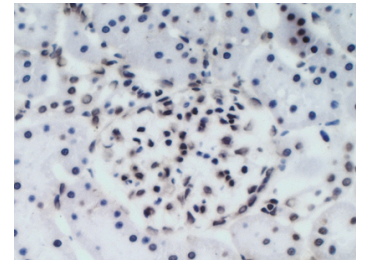

(C)

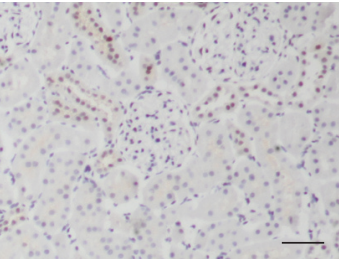

(B)

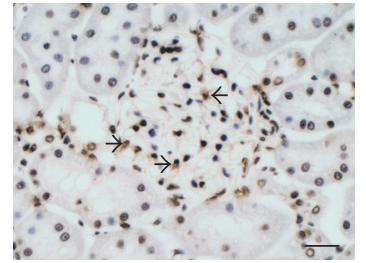

(D)

(a)
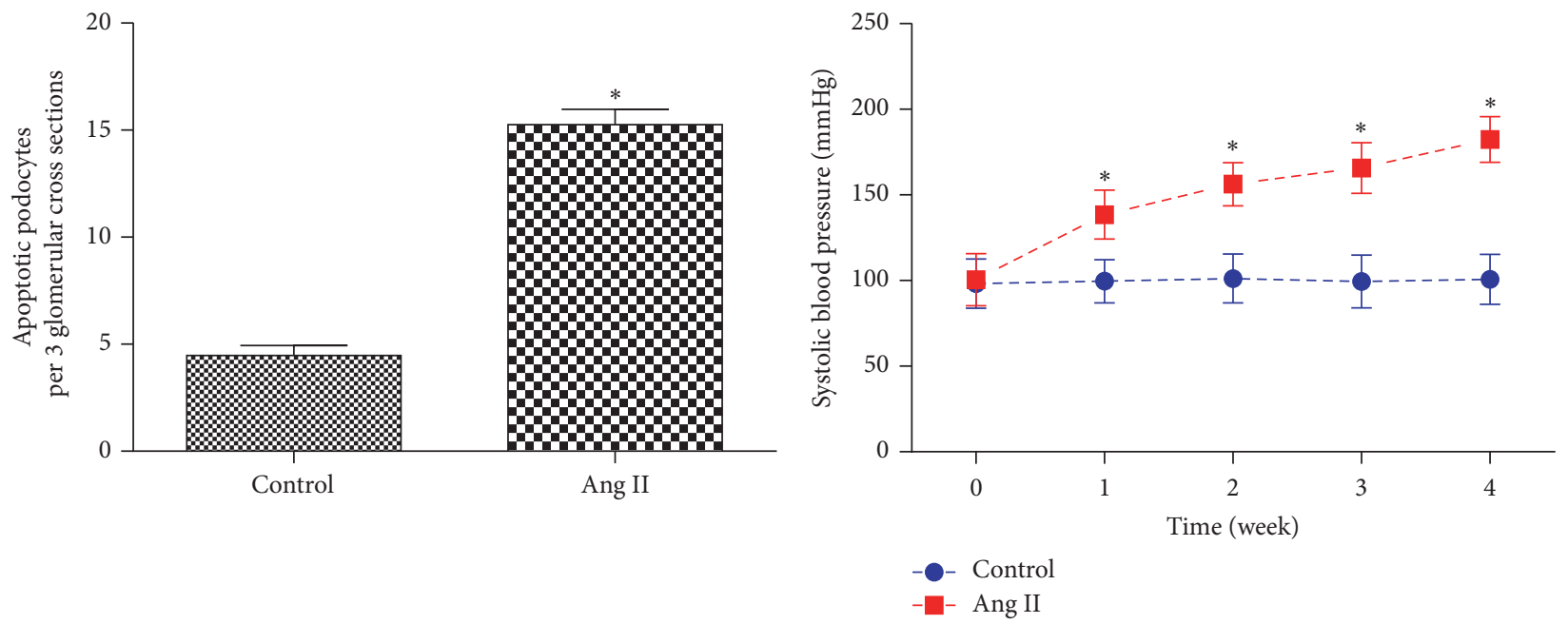

(c)

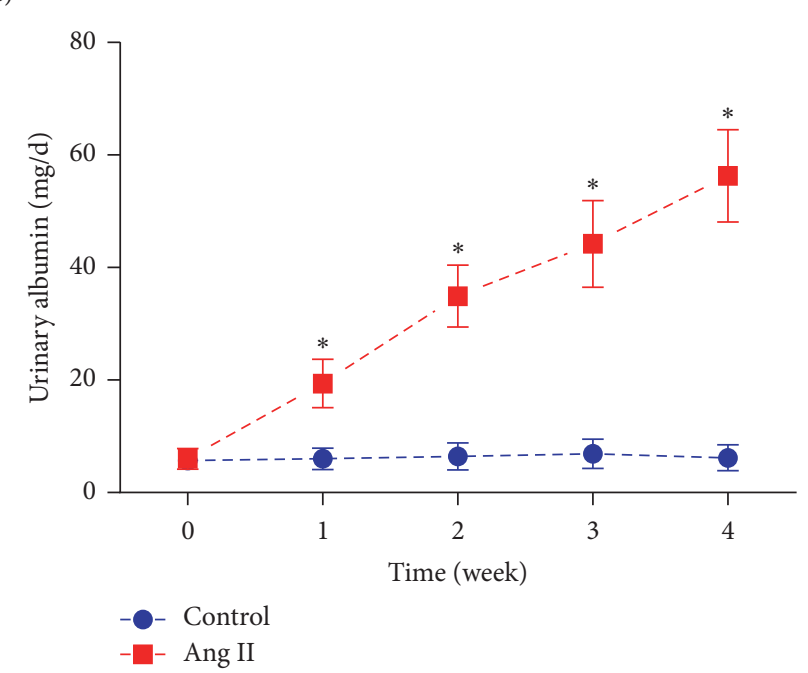

(d)

(e)

FIGURE 1: Clinical and pathological changes. (a) Glomerular pathological changes with PAS staining (original magnification $\times 200, \times 400$ ); (A) control group in week 4; (B) Ang II-infused group in week 4. Scale bars, $50 \mu \mathrm{m}$. (C) Control group in week 4; (D) Ang II-infused group in week 4. Scale bars, $20 \mu \mathrm{m}(n=6)$. (b) Apoptotic podocytes of rat kidneys were assessed by TUNEL staining (original magnification $\times 100$, $\times 400$ ); (A) control group in week 4; (B) Ang II-infused group in week 4; scale bars, $100 \mu \mathrm{m}$. (C) Control group in week 4; (D) Ang II-infused group in week 4 . The black arrows indicate apoptotic podocytes. Scale bars, $20 \mu \mathrm{m}(n=6)$. (c) Quantification of apoptotic podocytes. (d) Systolic blood pressure in weeks $1,2,3$, and 4 . (e) $24 \mathrm{~h}$ urinary albumin in weeks $1,2,3$, and $4 .{ }^{*} \mathrm{P}<0.05$ versus control group. 
DAPI

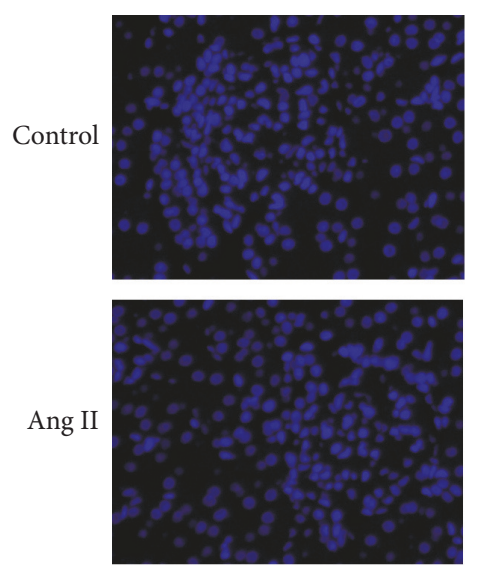

DAPI
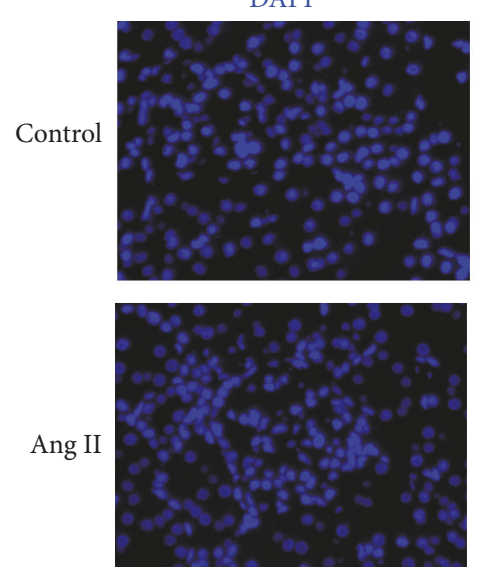

DAPI
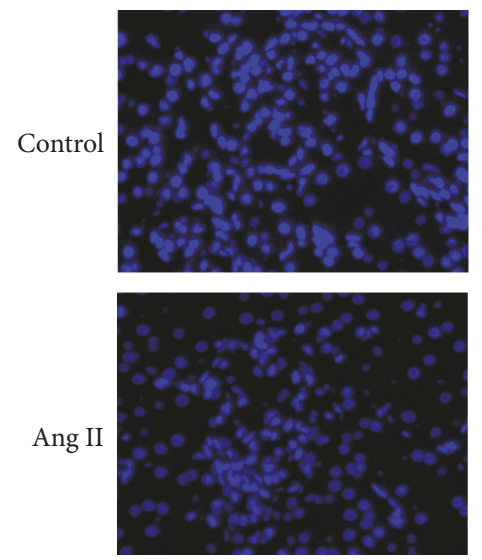

Dab1
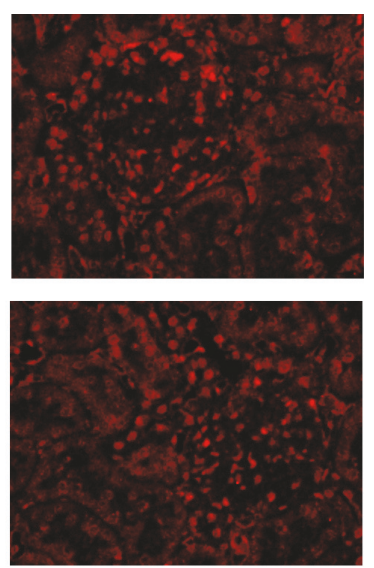

p-Dab1
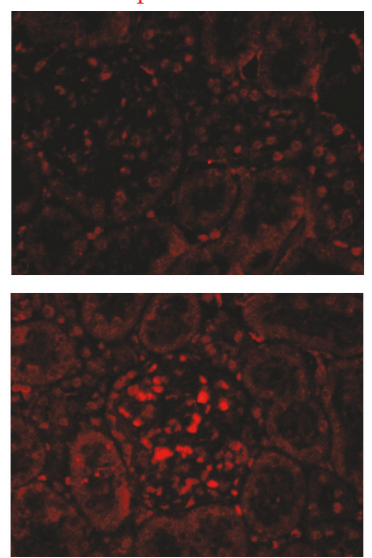

Reelin
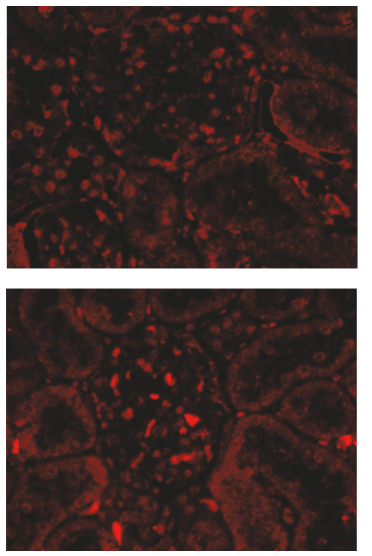

Nephrin
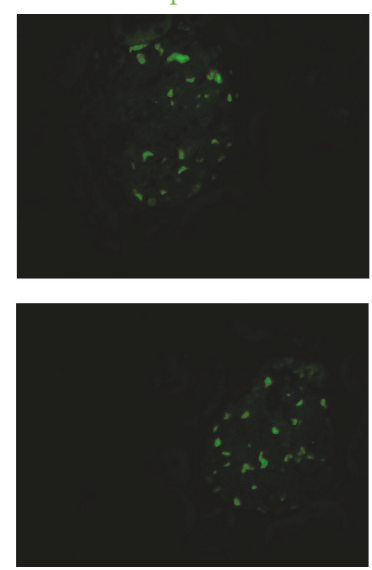

Nephrin
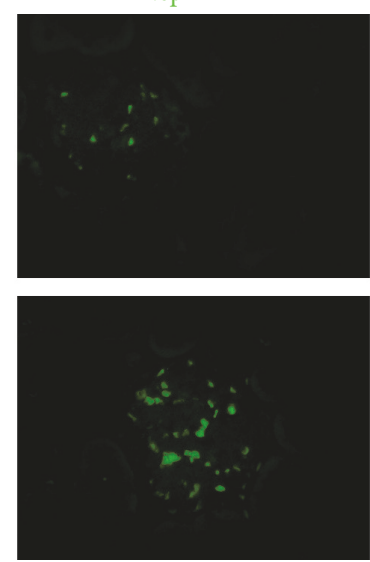

Nephrin
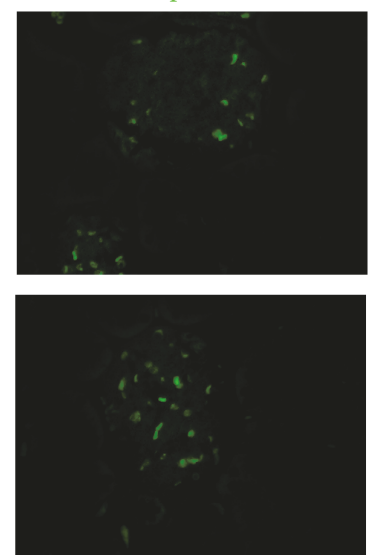

(a)
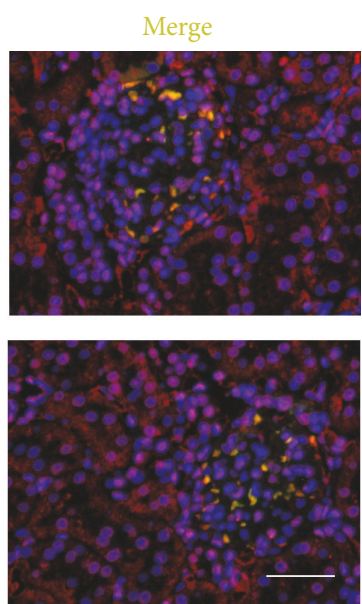

Merge
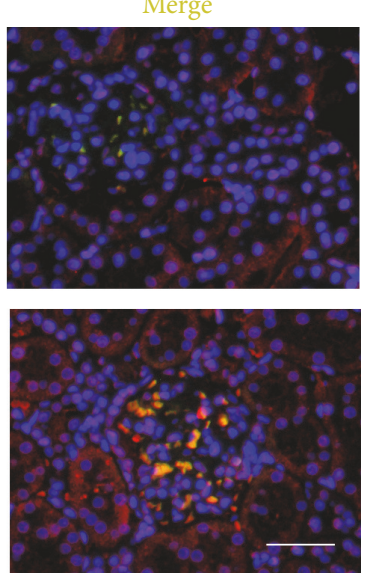

Merge
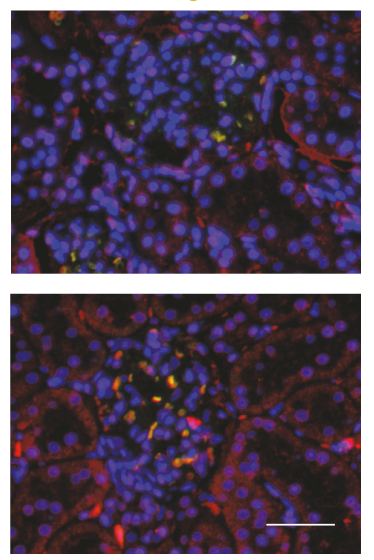

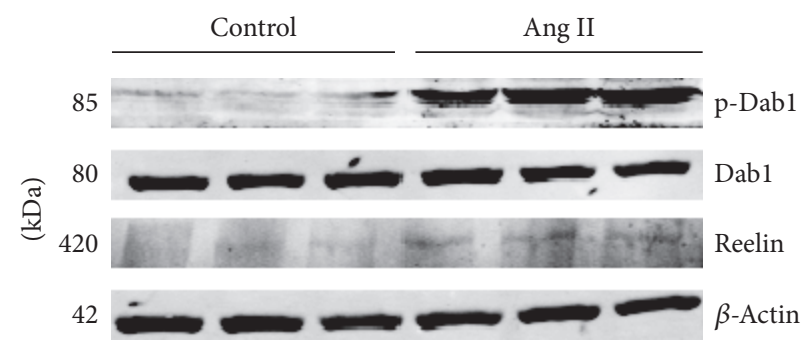

(b)

FIGURE 2: Continued. 


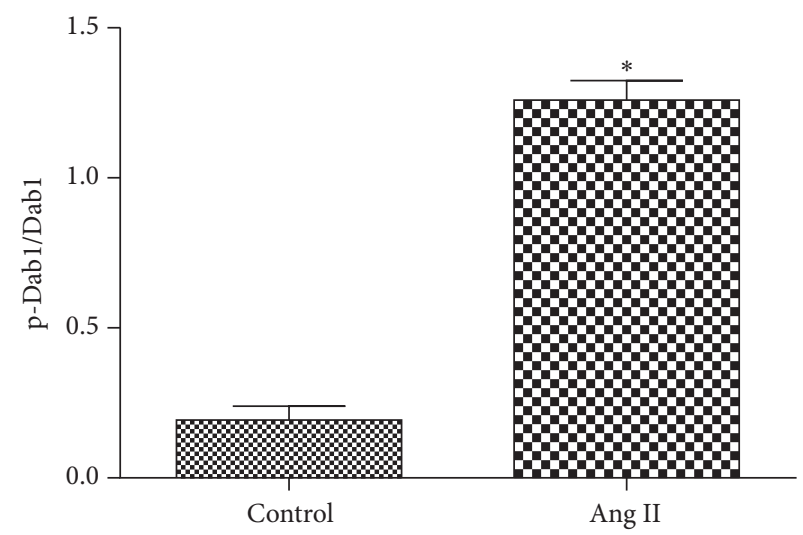

(c)

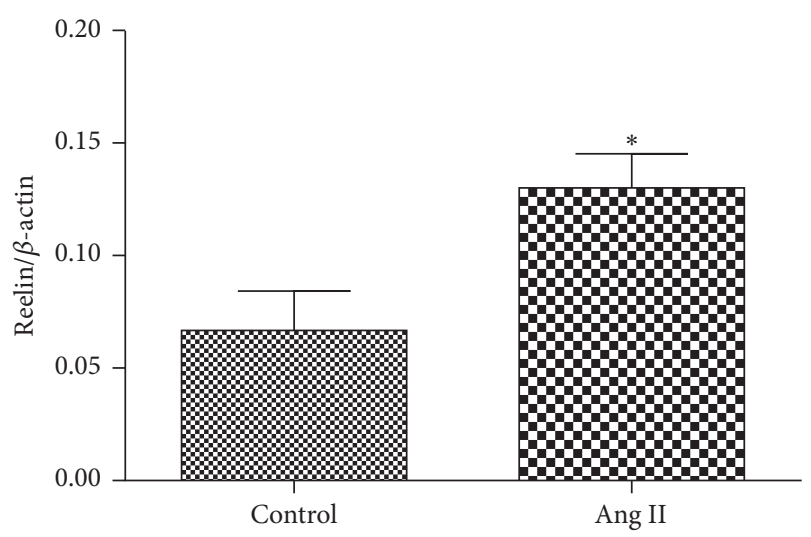

(d)

FIgURE 2: Ang II increases reelin/Dabl expression in kidney. (a) Double immunofluorescence staining for podocyte marker nephrin and various molecules (Dabl, phospho-Dabl, and reelin) in rat kidney (original magnification $\times 200$ ); scale bars, $50 \mu \mathrm{m}$. $(n=6)$. (b) Western blotting detected the expressions of phospho-Dabl, Dabl, and reelin in kidney of rats infused by Ang II. (c, d) Quantitative analysis of phosphoDab1 and reelin expression. ${ }^{*} P<0.05$ versus control group.

2.7. Statistical Analysis. Data were presented as means \pm SD and analyzed with SPSS 17.0. Student's $t$ test or oneway analysis of variance was used to compare differences between groups. A $P$ value $<0.05$ was considered statistically significant.

\section{Results}

3.1. Effect of Ang II on Kidney Injury. Figure 1(a) exhibits that the manifestation of glomerulus in Ang II infused rats showed cellular proliferation and accumulation of extracellular matrix. The characterization of glomerular damage was consistent with our past observation [10]. Moreover, the percentage of apoptotic podocytes in Ang II-infused rats was significantly increased compared with the control group (Figures 1(b) and 1(c)). Figures 1(d) and 1(e) show that systolic blood pressure and urinary albumin level were increased in Ang II-infused rats compared with controls in weeks 1, 2, 3, and 4 .

\subsection{Effect of Ang II on Reelin/Dabl Expression in Kidney.} Figure 2(a) demonstrates that the reelin expression and phosphorylated Dabl level in Ang II-infused rats were higher than those in the control group. The results of western blotting were consistent with double immunofluorescence assay (Figures 2(b)-2(d)).

\subsection{Effect of Ang II on Reelin/Dab1 Expression in Podocytes} and Podocyte Apoptosis. To investigate the effect of Ang II on the in vitro expression of reelin/Dabl, we assessed the reelin/Dabl expression in cultured podocytes. Podocytes were treated with $10^{-6} \mathrm{M}$ Ang II for several time points $(0,3$, 6,12 , and $24 \mathrm{~h}$ ) [10]. Ang II significantly increased the levels of reelin and phosphorylated Dabl in cultured podocytes in a time-dependent manner (Figures 3(a)-3(c)). Subsequently, immunofluorescence was performed to evaluate the distribution and expression of reelin and phosphorylated Dab1 in
Ang II-treated podocytes (Figure 3(d)). Expressions of bcl-2 and caspase-3 were determined to evaluate Ang II-induced podocyte apoptosis. Our data revealed that Ang II exposure significantly increased podocyte apoptosis (Figure 3(e)). The caspase-3 level was also increased after Ang II stimulation in vitro in a time-dependent manner (Figure 3(f)). Conversely, the bcl-2 expression was decreased after Ang II stimulation in podocytes in a time-dependent manner (Figure 3(f)).

3.4. Effect of Dabl on Ang II-Induced Podocyte Apoptosis. Podocytes were transfected with Dabl siRNA to evaluate the role of Dabl in Ang II-induced podocyte apoptosis. Figures 4(a) and 4(b) show that Dabl siRNA effectively reduced the phospho-Dabl and Dabl expression in podocytes. Western blotting was used to assess podocyte apoptosis. Conversely, the siRNA-triggered Dabl downregulation prevented Ang IIinduced podocyte apoptosis (Figures 4(c) and 4(d)), indicating that Dab1 was involved in the signaling pathways of Ang II-induced podocyte apoptosis.

3.5. Effect of Dab1 siRNA on Ang II-Induced p38 and ERK Pathways in Podocytes. It is well known that p38 and ERK pathways participate in regulation of cellular proliferation, differentiation, apoptosis, and so on [17]. Therefore, we evaluated whether p38 and ERK were involved in Ang IIinduced podocyte apoptosis when Dabl was suppressed by siRNA (Figure 5(a)). Figure 5(b) reveals that Ang II promoted phosphorylated p38 level, but the induced phosphorylation of p38 was diminished by Dabl siRNA. However, the ERK expression was not affected by Dabl siRNA (Figure 5(c)). Moreover, knockdown of Dab1 decreased the Ang II-induced upregulation of reelin (Figure 5(d)).

\section{Discussion}

In the present study, we found that Ang II played an important role in the process of proteinuria and podocyte 


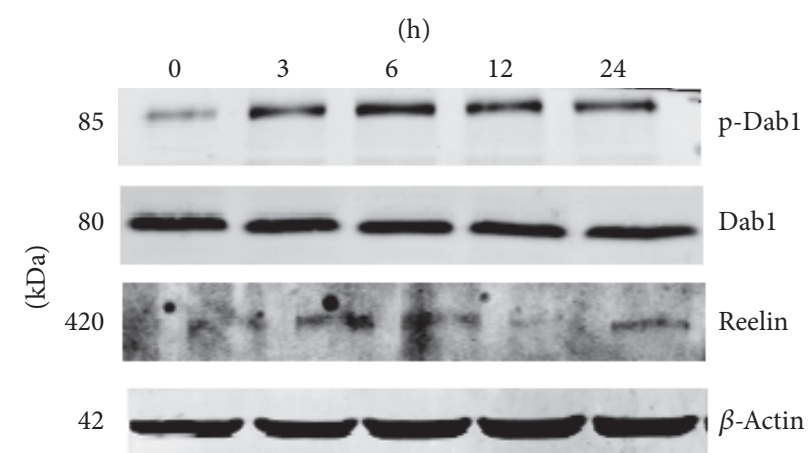

(a)

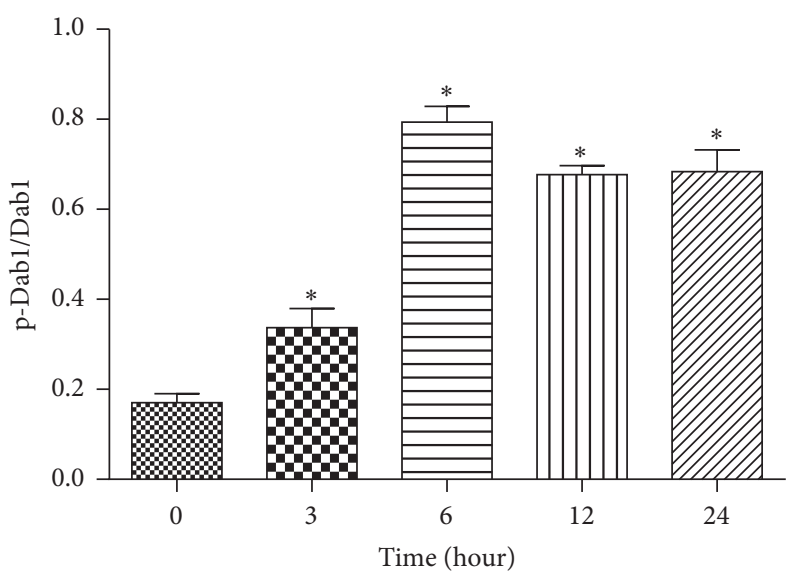

(b)

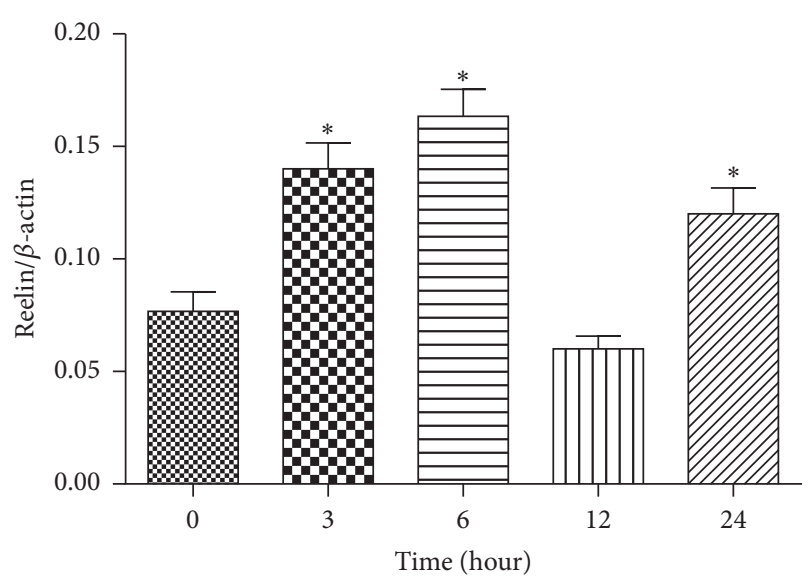

(c)

DAPI

Dab1

Merge
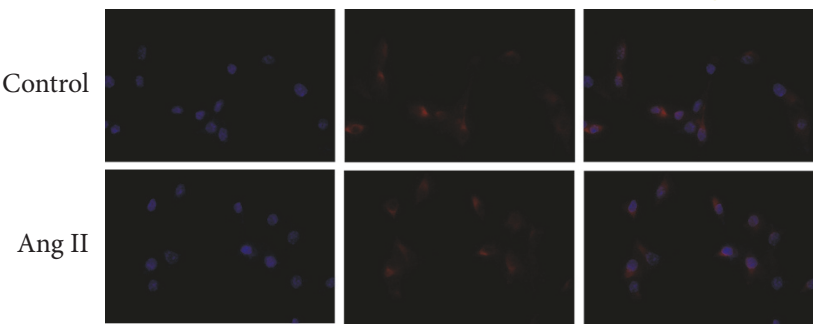

DAPI
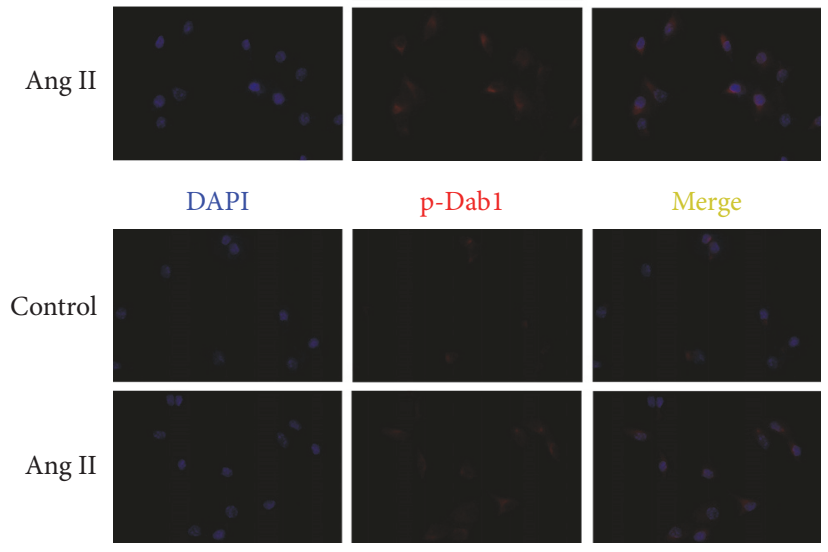

p-Dab1

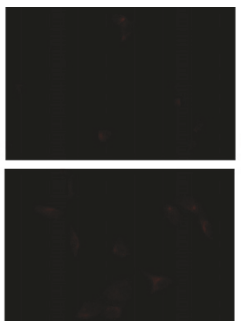

Merge

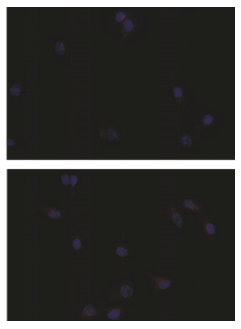

DAPI

Reelin

Merge
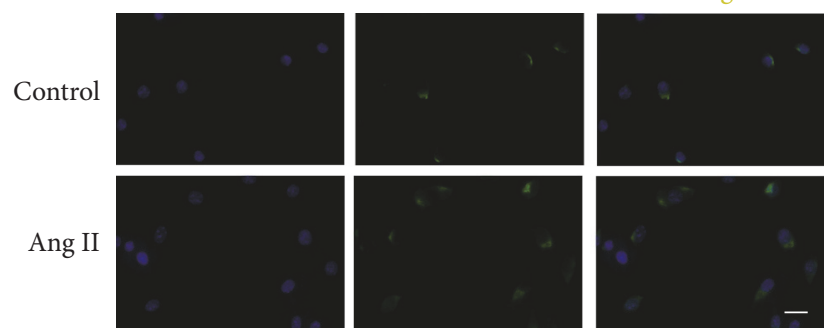

(d)

Figure 3: Continued. 


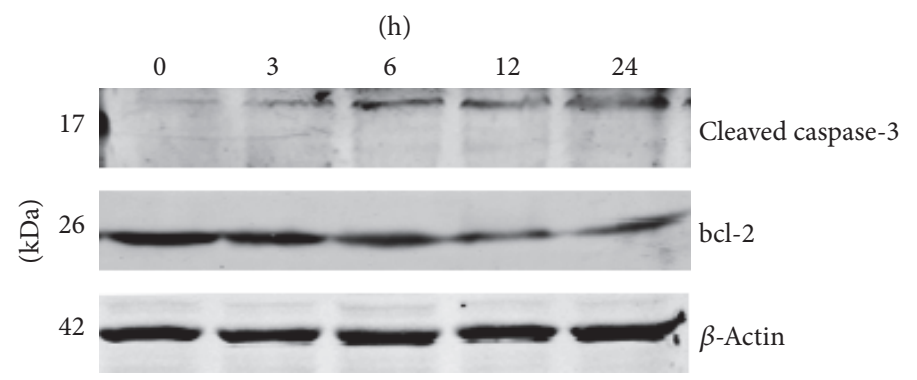

(e)

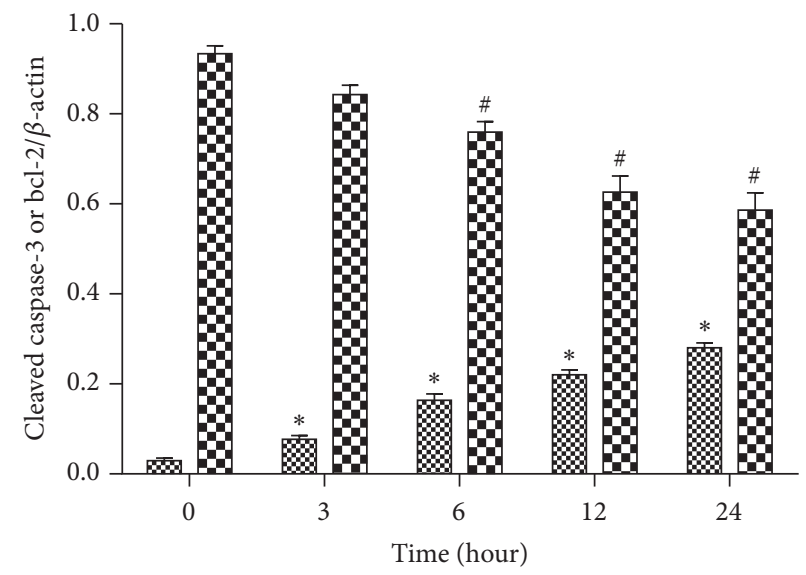

88 Cleaved caspase-3

Bol-2

(f)

Figure 3: Ang II increases reelin/Dab1 expression in cultured podocytes and induces podocytes apoptosis. (a) Western blotting detected the expressions of phospho-Dab1, Dabl, and reelin in cultured podocytes treated by Ang II $\left(10^{-6} \mathrm{M}\right)$ at various time points. (b, c) Quantitative analysis of phospho-Dab1 and reelin expression. (d) Immunofluorescence staining of Dab1, phospho-Dabl, and reelin in cultured podocytes stimulated by Ang II $\left(10^{-6} \mathrm{M}\right.$ ) for $6 \mathrm{~h}$ (original magnification $\times 400$ ); scale bars, $20 \mu \mathrm{m}$. (e) Western blotting detected the expressions of cleaved caspase- 3 and bcl- 2 in cultured podocytes treated by Ang II $\left(10^{-6} \mathrm{M}\right)$ at various time points. (f) Quantitative analysis of cleaved caspase- 3 and bcl-2 expressions. ${ }^{*} P<0.05$ versus control group. ${ }^{\sharp} P<0.05$ versus control group.

injury. Our previous studies have confirmed the effects of Ang II on podocyte apoptosis and albuminuria [10-12], but the underlying mechanism remains largely unexplored. Here, we reported that Dab1 contributed to Ang II-induced podocyte apoptosis via p38 signaling pathway.

As a secreted glycoprotein, reelin is expressed in CajalRetzius cells and granule cells in the cerebellum and olfactory bulb [18, 19]. Reelin plays an important role in the development and maturation of central nervous system, such as neuronal migration, neuronal excitability, and dendritic morphology. Binding of reelin to its receptors VLDLR and/or ApoER2 induces the phosphorylation of adaptor protein Dab1 by the Src family kinases Src and Fyn [20, 21]. Therefore, reelin regulates neuronal development through Dabl activation. However, the reelin/Dabl signaling pathway and its role in tissues other than the brain are still poorly understood. In the present study, reelin and Dabl were detected in kidney of rats and cultured mouse podocytes. Moreover, Ang II facilitated the reelin expression and Dabl phosphorylation in vivo and in vitro, indicating that reelin and Dab1 were involved in kidney disease and podocyte injury. Consistent with the previous studies, Ang II induced apoptosis of cultured podocytes in a time-dependent manner. However, the siRNA-triggered Dabl downregulation inhibited Ang IIinduced podocyte apoptosis. These results suggested that Dabl participated in the signaling pathways in the process of Ang II-induced podocyte apoptosis.

In neural development, phospho-Dabl promotes neuronal migration and regulates neuronal polarization via activating downstream Crk-C3G-Rapl-cadherin and PI3K-Akt signaling pathways [14]. Crk pathway regulates cytoskeleton, and PI3K-Akt pathway modulates cell survival, proliferation, metabolism, and angiogenesis. Dabl mutant mice exhibit ataxia, tremors, a reeling gait, and neurons aberrantly positioned in laminated brain structures [22], demonstrating that Dab1 phosphorylation is critical for initiation of intracellular signaling. Recently, Yang et al. [23] have reported that binding of activated protein $\mathrm{C}$ to ApoER2 activates Dab1-PI3K-Akt signaling in U937 cells. Furthermore, Dab1 has been implicated in the regulation of cholesterol efflux in RAW 264.7 mouse macrophage cell line [24]. VázquezCarretero et al. have reported that Dab1 participates in 



\&ab1

DOP $\mathrm{p}-\mathrm{Dab} 1$

(a)

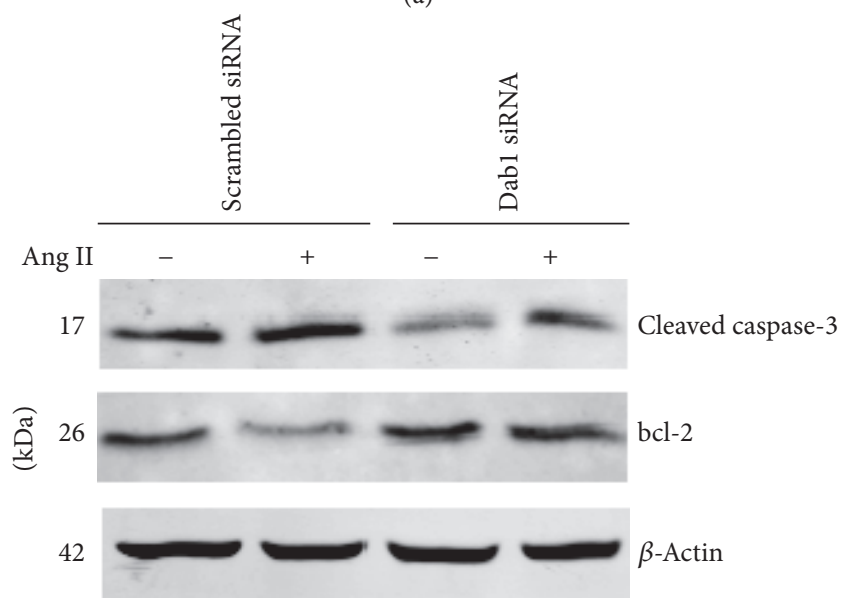

(c)

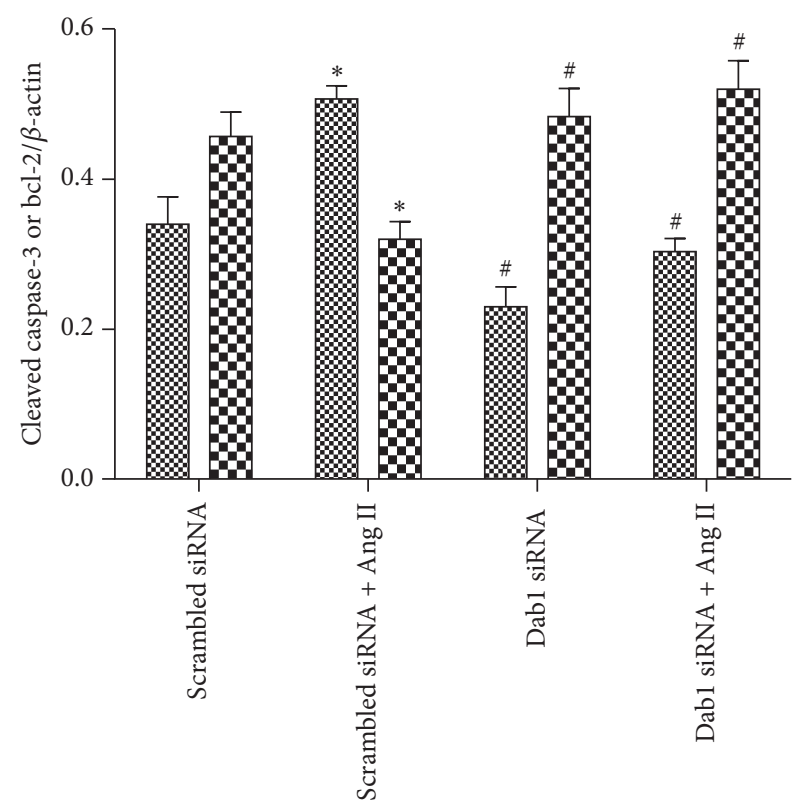

8. Cleaved caspase-3

bol-2

(b)

c)

(d)

FIGURE 4: Knockdown of Dab1 diminishes Ang II-induced podocyte apoptosis. (a) Western blotting detected phospho-Dabl and Dab1 expression in podocytes transfected with Dabl siRNA. Scrambled siRNA represents a nonspecific nonsilencing siRNA. (b) Quantitative analysis of phospho-Dab1 and Dab1 expression. (c) Western blotting detected expressions of cleaved caspase-3 and bcl-2 in podocytes transfected with Dab1 siRNA in the presence or absence of Ang II. (d) Quantitative analysis of cleaved caspase-3 and bcl-2 expressions. ${ }^{*} P<0.05$ versus control group. ${ }^{* *} P<0.01$ versus control group. ${ }^{\#} P<0.05$ versus podocytes transfected with scrambled siRNA and treated with Ang II. 


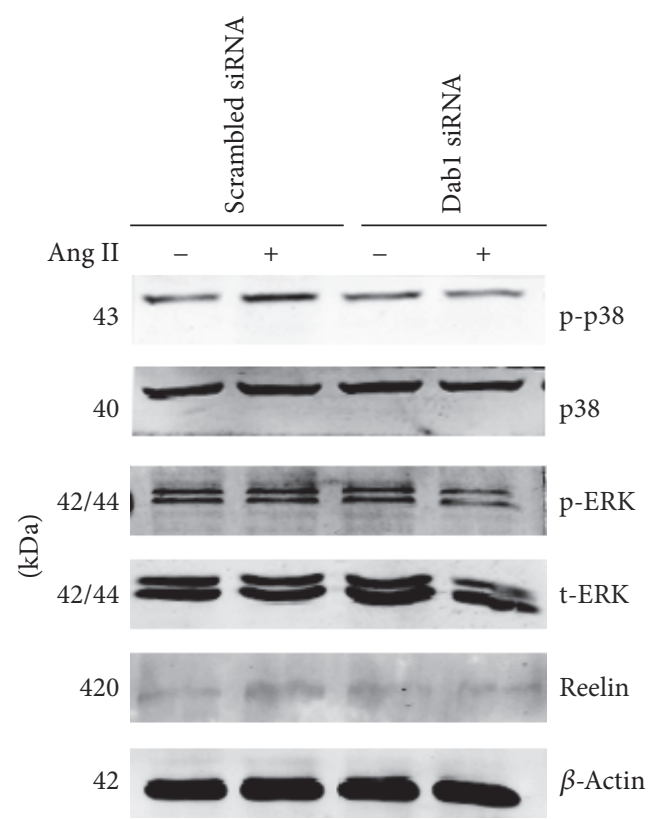

(a)

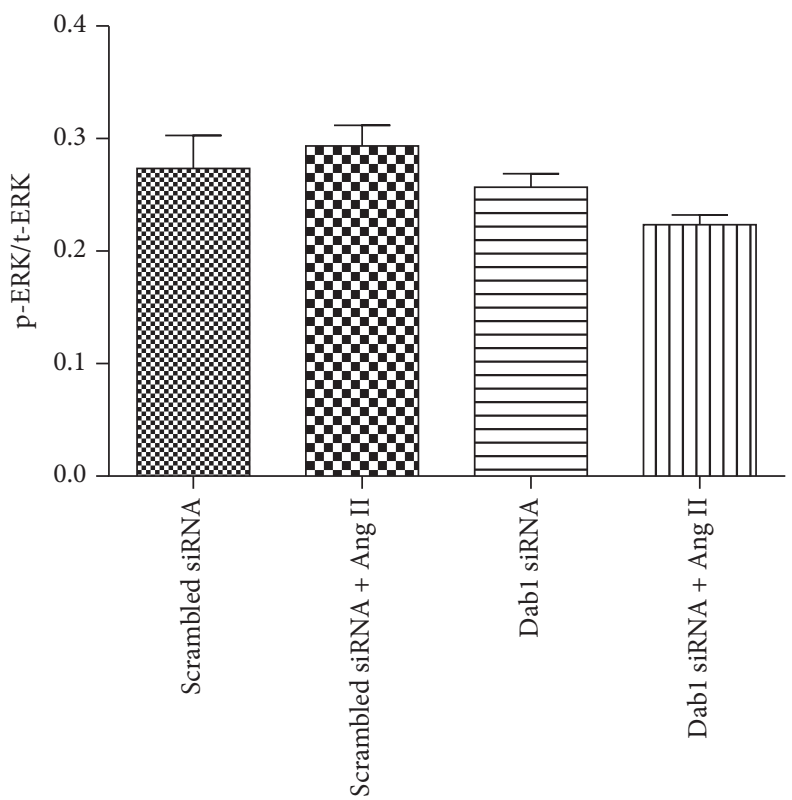

(c)

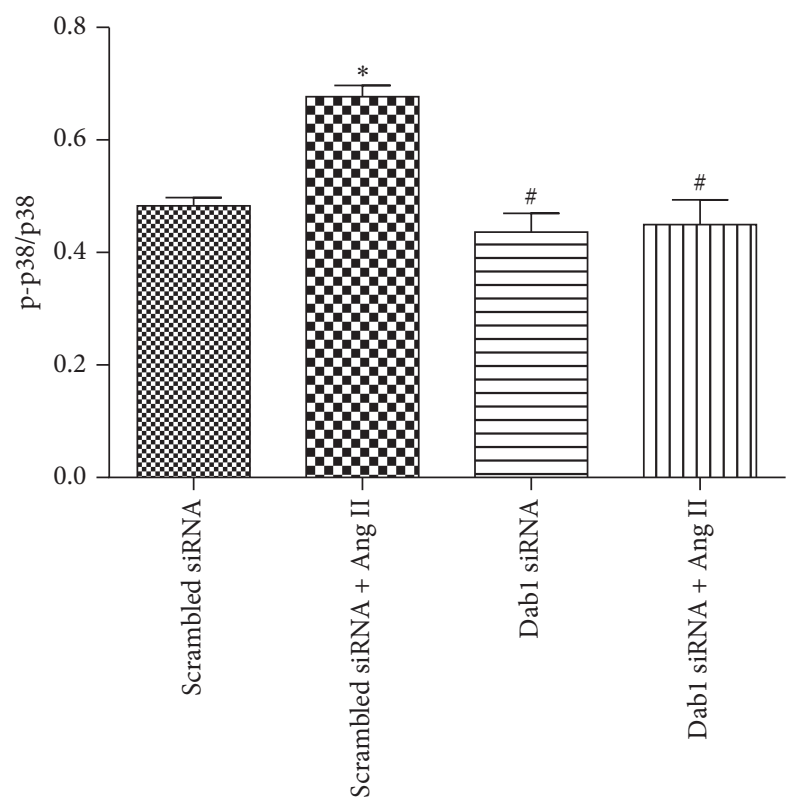

(b)

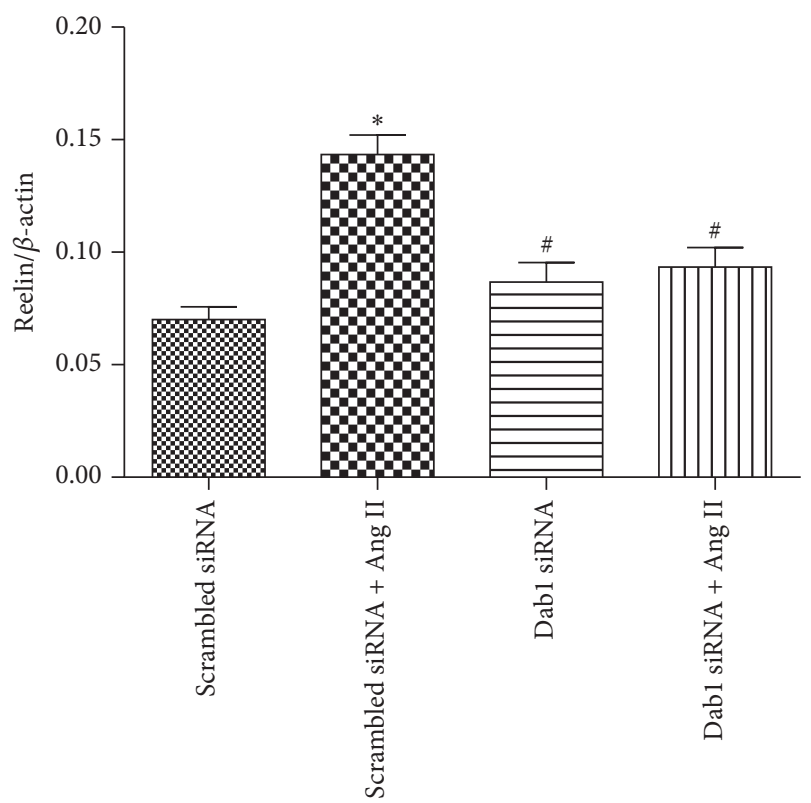

(d)

FIGURE 5: Knockdown of Dabl affects the MAPK signaling pathway and reelin expression in Ang II-treated podocytes. (a) Western blotting detected phospho-P38, phospho-ERK, and reelin expression in the podocytes. (b-d) Quantitative analysis of phospho-P38, phospho-ERK, and reelin expression. ${ }^{*} P<0.05$ versus control group. ${ }^{\#} P<0.05$ versus podocytes transfected with scrambled siRNA and treated with Ang II. 
regulation of intestinal crypt/villus unit dynamics [25]. In addition, Dabl is involved in regulation of mammary gland development, cartilage and tendon differentiation, odontogenesis, and granulosa cell proliferation in chicken follicle [26-30].

We investigated the effects of Dabl on the MAPK signaling pathway, which plays a vital role in regulation of cellular proliferation, differentiation, and apoptosis [17], because Dabl can regulate cell survival and proliferation. In our study, knockdown of Dabl could inhibit proapoptotic effects of Ang II. Subsequently, we explored the p38 and ERK pathway related to cellular apoptosis and proliferation of podocytes. Figure 5 reveals that Dabl downregulation inhibited phosphorylation of $\mathrm{p} 38$, whereas phosphorylation of ERK was not affected, indicating that Dabl was involved in Ang II-induced podocyte apoptosis via p38 pathway. Our previous study has confirmed that Ang II induces podocyte apoptosis through p38 MAPK pathway [31]. Recently, Gu et al. [32] have found that olmesartan decreases albuminuria in diabetic nephropathy through inhibiting Ang II/p38/SIRT1caused podocyte apoptosis. Interestingly, the reelin expression was decreased in podocytes when Dabl was knocked down. We did not detect the reelin expression in culture medium (data not shown). It is necessary to explore how Dabl affected the reelin expression in podocytes in future studies.

\section{Conclusions}

Our data showed that Dab1 and reelin could be expressed in rat kidney and cultured mouse podocytes. Podocytes and rats treated with Ang II displayed increased phosphoDabl and reelin expression. Ang II could induce apoptosis of cultured podocytes, and the knockdown of Dab1 by specific siRNA inhibited proapoptotic effects of Ang II. Furthermore, Dabl contributed to Ang II-induced podocyte apoptosis via p38 signaling. Therefore, the Dabl-p38 signaling pathway might be a novel candidate therapeutic target in order to decrease podocyte apoptosis in chronic kidney diseases.

\section{Conflicts of Interest}

The authors declare that there are no conflicts of interest.

\section{Authors' Contributions}

Zhao Gao and Xinghua Chen contributed equally to this work.

\section{Acknowledgments}

This work was financially supported by grants from the National Science Foundation of China (81500516 to Xinghua Chen and 81570617 to Guohua Ding) and the Natural Science Foundation of Hubei Province (2015CFB347 to Xinghua Chen).

\section{References}

[1] S. J. Shankland, "The podocyte's response to injury: role in proteinuria and glomerulosclerosis," Kidney International, vol. 69, no. 12, pp. 2131-2147, 2006.

[2] A. Greka and P. Mundel, "Cell biology and pathology of podocytes," Annual Review of Physiology, vol. 74, pp. 299-323, 2012.

[3] Lai M. A., K. W. Young, and U. Andag, "Targeting the podocyte to treat glomerular kidney disease," Drug Discovery Today, vol. 20, no. 10, pp. 1228-1234, 2015.

[4] P. Mundel and J. Reiser, "Proteinuria: an enzymatic disease of the podocyte," Kidney International, vol. 77, no. 7, pp. 571-580, 2010.

[5] K. N. Campbell, L. Raij, and P. Mundel, "Role of angiotensin II in the development of nephropathy and podocytopathy of diabetes," Current Diabetes Reviews, vol. 7, no. 1, pp. 3-7, 2011.

[6] P. T. Brinkkoetter, C. Ising, and T. Benzing, "The role of the podocyte in albumin filtration," Nature Reviews Nephrology, vol. 9, no. 6, pp. 328-336, 2013.

[7] F. Cellesi, M. Li, and M. P. Rastaldi, "Podocyte injury and repair mechanisms," Current Opinion in Nephrology and Hypertension, vol. 24, no. 3, pp. 239-244, 2015.

[8] J. L. Zhuo and X. C. Li, "New insights and perspectives on intrarenal renin-angiotensin system: Focus on intracrine/ intracellular angiotensin II," Peptides, vol. 32, no. 7, pp. 15511565, 2011.

[9] C. Rüster and G. Wolf, "Renin-angiotensin-aldosterone system and progression of renal disease," Journal of the American Society of Nephrology, vol. 17, no. 11, pp. 2985-2991, 2006.

[10] Z. Ren, W. Liang, C. Chen, H. Yang, P. C. Singhal, and G. Ding, "Angiotensin II induces nephrin dephosphorylation and podocyte injury: role of caveolin-1," Cellular Signalling, vol. 24, no. 2, pp. 443-450, 2012.

[11] X. H. Chen, Z. L. Ren, W. Liang et al., "c-Abl mediates angiotensin II-induced apoptosis in podocytes," Journal of Molecular Histology, vol. 44, no. 5, pp. 597-608, 2013.

[12] Q. Yang, Y. Ma, Y. Liu et al., "Angiotensin II down-regulates nephrin-akt signaling and induces podocyte injury: Role of cAbl," Molecular Biology of the Cell, vol. 27, no. 1, pp. 197-208, 2016.

[13] Z. H. Gao and R. Godbout, "Reelin-Disabled-1 signaling in neuronal migration: splicing takes the stage," Cellular and Molecular Life Sciences, vol. 70, no. 13, pp. 2319-2329, 2013.

[14] A. M. Stranahan, J. R. Erion, and M. Wosiski-Kuhn, "Reelin signaling in development, maintenance, and plasticity of neural networks," Ageing Research Reviews, vol. 12, no. 3, pp. 815-822, 2013.

[15] K. E. White and R. W. Bilous, "Estimation of podocyte number: A comparison of methods," Kidney International, vol. 66, no. 2, pp. 663-667, 2004.

[16] S. B. Nicholas, J. M. Basgen, and S. Sinha, "Using stereologic techniques for podocyte counting in the mouse: Shifting the paradigm," American Journal of Nephrology, vol. 33, no. 1, pp. $1-7,2011$.

[17] E. K. Kim and E.-J. Choi, "Compromised MAPK signaling in human diseases: an update," Archives of Toxicology, vol. 89, no. 6, pp. 867-882, 2015.

[18] E. Martin-Lopez, A. Blanchart, J. A. de Carlos, and L. LópezMascaraque, "Dabl (disable homolog-1) reelin adaptor protein is overexpressed in the olfactory bulb at early postnatal stages," PLoS ONE, vol. 6, no. 10, Article ID e26673, 2011. 
[19] G. H. Lee and G. D'Arcangelo, "New insights into reelinmediated signaling pathways," Frontiers in Cellular Neuroscience, vol. 10, no. MAY, article no. 122, 2016.

[20] F. M. Ranaivoson, S. von Daake, and D. Comoletti, "Structural insights into reelin function: Present and future," Frontiers in Cellular Neuroscience, vol. 10, no. MAY, article no. 137, 2016.

[21] H. H. Bock and P. May, "Canonical and non-canonical reelin signaling," Frontiers in Cellular Neuroscience, vol. 10, article no. $166,2016$.

[22] M. Trommsdorff, M. Gotthardt, T. Hiesberger et al., "Reeler/ disabled-like disruption of neuronal migration in knockout mice lacking the VLDL receptor and ApoE receptor 2," Cell, vol. 97, no. 6, pp. 689-701, 1999.

[23] X. V. Yang, Y. Banerjee, J. A. Fernandez et al., "Activated protein C ligation of ApoER2 (LRP8) causes Dabl-dependent signaling in U937 cells," Proceedings of the National Academy of Sciences, vol. 106, no. 1, pp. 274-279, 2009.

[24] X. Chen, Z. Guo, E. U. Okoro et al., "Up-regulation of ATP binding cassette transporter A1 expression by very low density lipoprotein receptor and apolipoprotein E receptor 2," Journal of Biological Chemistry, vol. 287, no. 6, pp. 3751-3759, 2012.

[25] M. D. Vázquez-Carretero, P. García-Miranda, M. L. Calonge, M. J. Peral, and A. A. Ilundain, "Dab1 and reelin participate in a common signal pathway that controls intestinal crypt/villus unit dynamics," Biology of the Cell, vol. 106, no. 3, pp. 83-96, 2014.

[26] E. Khialeeva, T. F. Lane, and E. M. Carpenter, "Disruption of reelin signaling alters mammary gland morphogenesis," Development, vol. 138, no. 4, pp. 767-776, 2011.

[27] M. J. Díaz-Mendoza, C. I. Lorda-Diez, J. A. Montero, J. A. García-Porrero, and J. M. Hurlé, "Interdigital cell death in the embryonic limb is associated with depletion of Reelin in the extracellular matrix," Cell Death and Disease, vol. 4, no. 9, article no. e800, 2013.

[28] M. J. Diaz-Mendoza, C. I. Lorda-Diez, J. A. Montero, J. A. Garcia-Porrero, and J. M. Hurle, "Reelin/DAB-1 signaling in the embryonic limb regulates the chondrogenic differentiation of digit mesodermal progenitors," Journal of Cellular Physiology, vol. 229, no. 10, pp. 1397-1404, 2014.

[29] H. Magloire, M.-L. Couble, B. Thivichon-Prince, J.-C. Maurin, and F. Bleicher, "Odontoblast: A mechano-sensory cell," Journal of Experimental Zoology Part B: Molecular and Developmental Evolution, vol. 312, no. 5, pp. 416-424, 2009.

[30] C. Eresheim, C. Leeb, P. Buchegger, and J. Nimpf, "Signaling by the extracellular matrix protein reelin promotes granulosa cell proliferation in the chicken follicle," Journal of Biological Chemistry, vol. 289, no. 14, pp. 10182-10191, 2014.

[31] Y. Liu, W. Liang, Q. Yang et al., "IQGAP1 mediates Angiotensin ii-induced apoptosis of Podocytes via the ERK1/2 MAPK signaling pathway," American Journal of Nephrology, vol. 38, no. 5, pp. 430-444, 2013.

[32] J. Gu, M. Yang, N. Qi et al., "Olmesartan Prevents Microalbuminuria in $\mathrm{db} / \mathrm{db}$ Diabetic Mice Through Inhibition of Angiotensin II/p38/SIRT1-Induced Podocyte Apoptosis," Kidney and Blood Pressure Research, vol. 41, no. 6, pp. 848-864, 2016. 

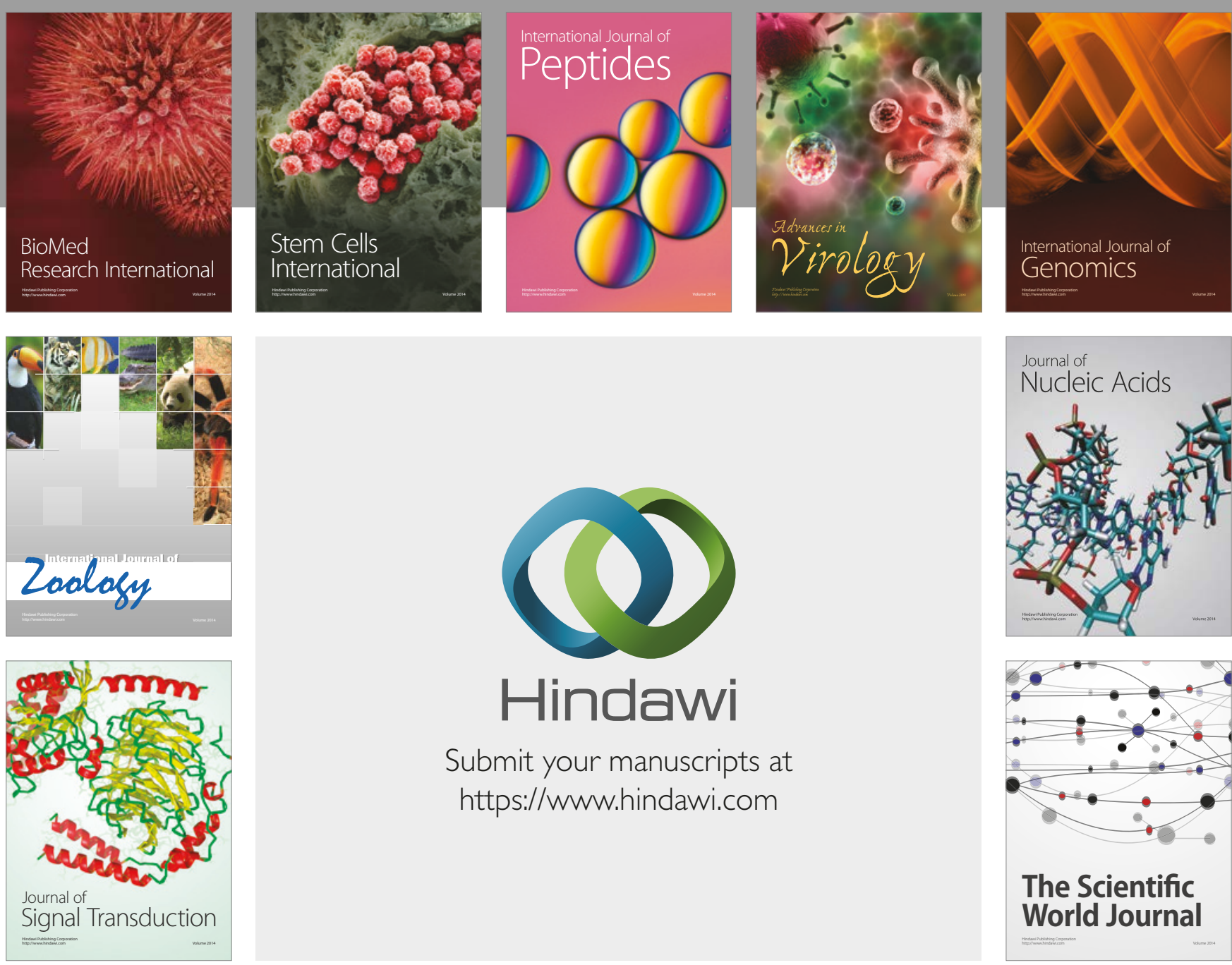

Submit your manuscripts at

https://www.hindawi.com
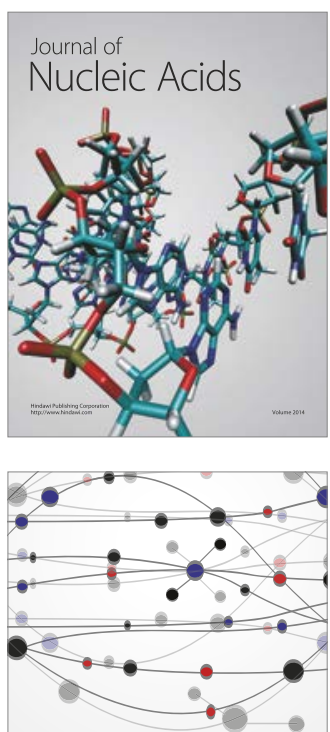

The Scientific World Journal

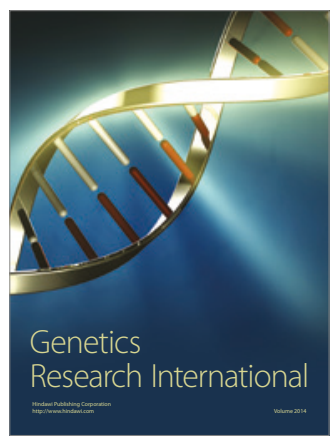

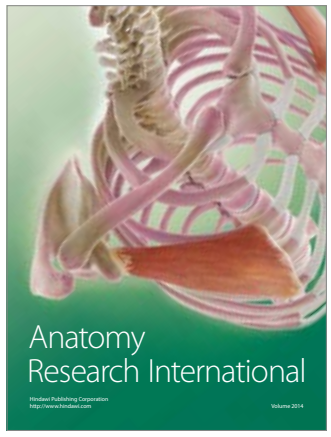

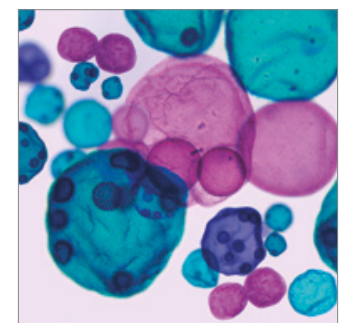

International Journal of Microbiology
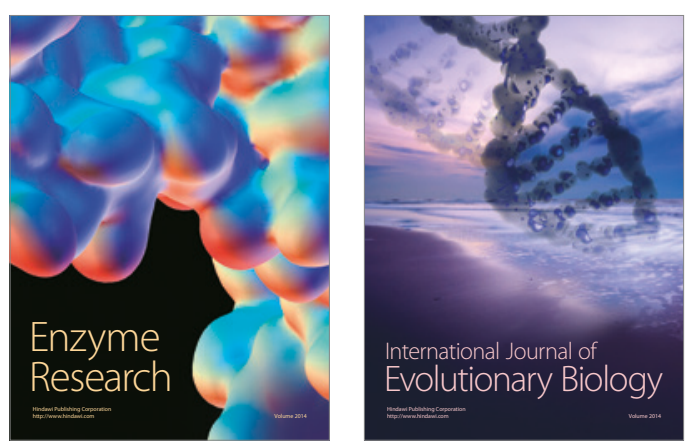
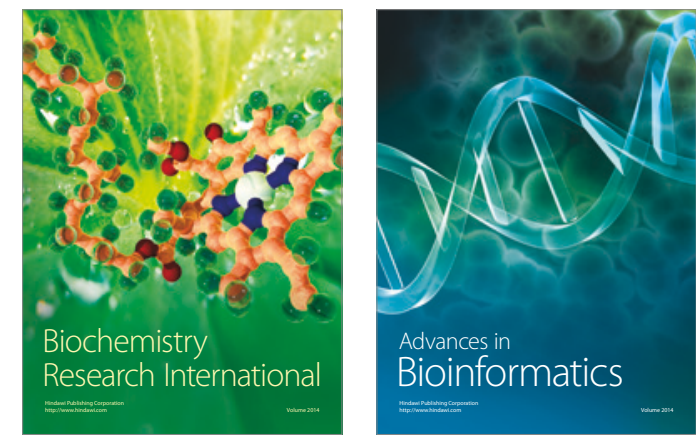

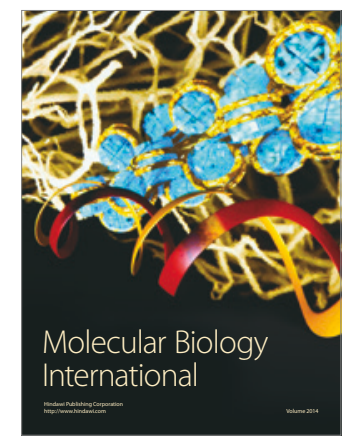

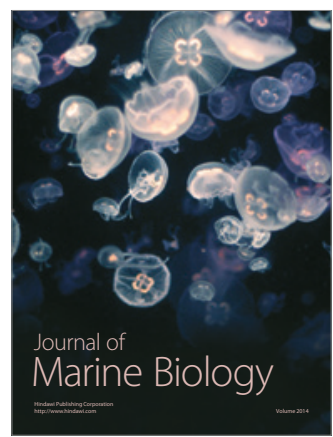

